\title{
Asymptotic symmetries of Maxwell Chern-Simons gravity with torsion
}

\author{
H. Adami ${ }^{1, \mathrm{a}}$, P. Concha ${ }^{2, \mathrm{~b}} \underset{\mathrm{C}}{ }$, E. Rodríguez $^{3, \mathrm{c}}$, H. R. Safari ${ }^{1, \mathrm{~d}}$ \\ ${ }^{1}$ School of Physics, Institute for Research in Fundamental Sciences (IPM), P.O. Box 19395-5531, Tehran, Iran \\ 2 Departamento de Matemática y Física Aplicadas, Universidad Católica de la Santísima Concepción, Alonso de Ribera 2850, Concepción, Chile \\ ${ }^{3}$ Departamento de Ciencias, Facultad de Artes Liberales, Universidad Adolfo Ibáñez, Viña del Mar, Chile
}

Received: 26 June 2020 / Accepted: 10 October 2020 / Published online: 19 October 2020

(C) The Author(s) 2020

\begin{abstract}
We present a three-dimensional Chern-Simons gravity based on a deformation of the Maxwell algebra. This symmetry allows introduction of a non-vanishing torsion to the Maxwell Chern-Simons theory, whose action recovers the Mielke-Baekler model for particular values of the coupling constants. By considering suitable boundary conditions, we show that the asymptotic symmetry is given by the $\widehat{\mathfrak{b m s}_{3}} \oplus \mathfrak{v i r}$ algebra with three independent central charges.
\end{abstract}

\section{Contents}

1 Introduction and motivations . . . . . . . . . . . 1

2 Maxwell Chern-Simons gravity theory with torsion 2

2.1 BMS-like solution . . . . . . . . . . . . 4

3 Asymptotic symmetries . . . . . . . . . . . . 5

3.1 Boundary conditions . . . . . . . . 5 5

3.2 Residual gauge transformations . . . . . . . . 6

3.3 Canonical surface charges and asymptotic symmetry algebra . . . . . . . . . 6

3.4 Change of basis . . . . . . . . . . . . 7

4 Discussion and outlook . . . . . . . . . . . 8

References . . . . . . . . . . . . . . . . 8

\section{Introduction and motivations}

Three-dimensional Chern-Simons (CS) gravity theories are considered as interesting toy models since they allow us to approach diverse aspects of the gravitational interaction and underlying laws of quantum gravity. Furthermore, they

\footnotetext{
a e-mail: hamed.adami@ipm.ir

b e-mail: patrick.concha@ucsc.cl (corresponding author)

c e-mail: evelyn.rodriguez@edu.uai.cl

de-mail: hrsafari@ipm.ir
}

share many properties with higher-dimensional gravity models which, in general, are more difficult to study. Three dimensional General Relativity (GR) with and without cosmological constant can be described through a CS action based on the AdS and Poincaré algebra, respectively [1-3]. Nowadays, there is a growing interest in exploring bigger symmetries in order to study more interesting and realistic physical models.

Well-known infinite-dimensional enhancements of the AdS and Poincaré symmetries, in three spacetime dimensions, are given respectively by the conformal and the $\mathfrak{b m s}_{3}$ algebras. A central extension of the two-dimensional conformal algebra, which can be written as two copies of the Virasoro algebra, appears as the asymptotic symmetry of threedimensional GR with negative cosmological constant [4]. In the asymptotically flat case, the three-dimensional version of the Bondi-Metzner-Sachs (BMS) algebra [5-8], denoted as $\mathfrak{b m s}_{3}$, corresponds to the asymptotic symmetry of GR [9]. $\mathfrak{b m s}_{3}$ can be alternatively obtained as a flat limit of the conformal one, in a similar way as the Poincaré symmetry appears as a vanishing cosmological constant limit of AdS. The study of richer boundary dynamics could offer a better understanding of the bulk/boundary duality beyond the AdS/CFT correspondence [10]. Thus, the exploration of new asymptotic symmetries of CS gravity theories based on enlarged global symmetries could be worth studying. In particular, extensions and generalizations of the conformal and the $\mathfrak{b m s}_{3}$ algebras have been subsequently developed in diverse contexts in [1134].

A particular extension and deformation of the Poincaré algebra is given by the Maxwell algebra. Such symmetry appears to describe a particle moving in a four-dimensional Minkowski background in presence of a constant electromagnetic field [35-37]. This algebra is characterized by the non-vanishing commutator of the four-momentum generator 
$P_{a}:$

$\left[P_{a}, P_{b}\right]=M_{a b}$

which is proportional to a new Abelian generator $M_{a b}$. The Maxwell algebra and its generalizations have been useful to recover standard GR without cosmological constant from CS and Born-Infeld gravity theories in a particular limit [3842]. In three spacetime dimensions, an invariant CS gravity action under Maxwell algebra has been introduced in [43] and different aspects of it has been studied [44-53]. As Poincaré symmetry, the Maxwell symmetry describes a three-dimensional gravity theory whose geometry is Riemannian and locally flat. However, the presence of an additional gauge field in the Maxwell case leads to new effects compared to GR. In particular, in [29], the authors have shown that the Maxwellian gravitational gauge field modifies not only the vacuum energy and angular momentum of the stationary configuration but also the asymptotic structure.

To accommodate a non-vanishing torsion to the Maxwell CS gravity theory it is necessary to deform the Maxwell algebra. Here, we show that a particular deformation of the Maxwell symmetry, which we refer to as "deformed Maxwell algebra", allows us to introduce not only a torsional but also a cosmological constant term along the Einstein-Hilbert term. Then, motivated by the recent results on the Maxwell algebra, we explore the effects of deforming the Maxwell symmetry both to the bulk and boundary dynamics. At the bulk level, we show that the invariant CS gravity action under the deformed Maxwell algebra reproduces the Maxwell field equations but with a non-vanishing torsion describing a RiemannCartan geometry. Interestingly, the CS action can be seen as a Maxwell version of a particular case of the MielkeBaekler (MB) gravity theory [54] which describes a threedimensional gravity model in presence of non-vanishing torsion. Further studies of the MB gravity have been subsequently developed in [55-65]. Here we explore the effects of having a non-vanishing torsion in Maxwell CS gravity at the level of the boundary dynamics. In particular, by considering suitable boundary conditions, we show that the asymptotic symmetry can be written as the $\widehat{\mathfrak{b m s}_{3}} \oplus$ vir algebra. This infinite-dimensional symmetry was recently obtained as a deformation of the infinite-dimensional enhancement of the Maxwell algebra, denoted as $\mathfrak{M a r}_{3}$ algebra [66]. We also show that the vanishing cosmological constant limit $\ell \rightarrow \infty$ can be applied not only at the CS gravity theory level but also at the asymptotic algebra, leading to the Maxwell CS gravity and its respective asymptotic symmetry previously introduced in [29].

The paper is organized as follows. In Sect. 2, we present the three-dimensional CS gravity theory which is invariant under a particular deformation of the Maxwell algebra. Furthermore, considering asymptotically flat geometries with null boundary, we discuss the BMS-like solution of the theory. We provide boundary conditions allowing a well-defined action principle. In Sect. 3, we show that the asymptotic symmetry algebra for the Maxwell CS gravity with torsion is given by an infinite enhancement of a deformed Maxwell algebra, which can be written as the direct sum $\widehat{\mathfrak{b m s}_{3}} \oplus$ vir. Finally, in Sect. 4 we discuss the obtained results and possible future developments.

Notation We adopt the same notation as $[30,66,67]$ for the algebras; for algebras we generically use "mathfrak" fonts, like $\mathfrak{v i r}, \mathfrak{b m s}_{3}$ and $\mathfrak{M a x}_{3}$. The centrally extended version of an algebra $\mathfrak{g}$ will be denoted by $\hat{\mathfrak{g}}$, e.g. Virasoro algebra $\mathfrak{v i r}=\widehat{\mathfrak{w i t t}}$.

\section{Maxwell Chern-Simons gravity theory with torsion}

Using the CS formalism, we present the three-dimensional gravity theory based on a particular deformation of the Maxwell algebra. Unlike the Maxwell case, such deformation leads to a non-vanishing torsion as equation of motion. The deformed Maxwell algebra is spanned by the generators $\left\{J_{a}, P_{a}, M_{a}\right\}$, which satisfy the following non-vanishing commutation relations:

$$
\begin{aligned}
& {\left[J_{a}, J_{b}\right]=\epsilon_{a b}{ }^{c} J_{c},} \\
& {\left[J_{a}, P_{b}\right]=\epsilon_{a b}{ }^{c} P_{c},} \\
& {\left[J_{a}, M_{b}\right]=\epsilon_{a b}{ }^{c} M_{c},} \\
& {\left[P_{a}, P_{b}\right]=\epsilon_{a b}{ }^{c}\left(M_{c}+\frac{1}{\ell} P_{c}\right),}
\end{aligned}
$$

where $\epsilon_{a b c}$ is the three-dimensional Levi-Civita tensor and $a, b=0,1,2$ are the Lorentz indices which are lowered and raised with the Minkowski metric $\eta_{a b}$. The $\ell$ parameter appearing in the last commutator is related to the cosmological constant $\Lambda$. Then, the vanishing cosmological constant limit $\ell \rightarrow \infty$ reproduces the Maxwell symmetry. Let us note that the Hietarinta-Maxwell algebra $[48,52,68]$ is recovered in the limit $\ell \rightarrow \infty$ when the role of the $P_{a}$ and $M_{a}$ generators is interchanged. One can see that $J_{a}$ and $P_{a}$ are not the generators of a Poincaré subalgebra. However, as it is pointed out in [66], (2.1) can be rewritten as the iso $(2,1) \oplus \mathfrak{s o}(2,1)$ algebra. This can be seen by a redefinition of the generators,

$$
\begin{aligned}
& L_{a} \equiv J_{a}-\ell P_{a}-\ell^{2} M_{a}, \\
& S_{a} \equiv \ell P_{a}+\ell^{2} M_{a}, \\
& T_{a} \equiv-\ell M_{a},
\end{aligned}
$$

where $L_{a}$ and $T_{a}$ are the respective generators of the iso $(2,1)$ algebra, while $S_{a}$ is a $\mathfrak{s o}(2,1)$ generator. Then, the Lie algebra 
(2.1) can be rewritten as

$$
\begin{aligned}
& {\left[L_{a}, L_{b}\right]=\epsilon_{a b}{ }^{c} L_{c},} \\
& {\left[L_{a}, T_{b}\right]=\epsilon_{a b}{ }^{c} T_{c},} \\
& {\left[S_{a}, S_{b}\right]=\epsilon_{a b}{ }^{c} S_{c} .}
\end{aligned}
$$

It is important to point out that (2.1) is not the unique way of deforming the Maxwell algebra. It is shown in [69] that the Maxwell algebra can be deformed into two different algebras: $\mathfrak{s o}(2,2) \oplus \mathfrak{s o}(2,1)$ and $\mathfrak{i s o}(2,1) \oplus \mathfrak{s o}(2,1)$. The former has been largely studied in $[31,44,70,71]$ whose asymptotic symmetry is described by three copies of the Virasoro algebra [31,32], while the latter has only been approached through a deformation process $[66,69]$. In the present work, using the basis $\left\{J_{a}, P_{a}, M_{a}\right\}$, we find asymptotic symmetry of the CS gravity theory based on the $i \mathfrak{s o}(2,1) \oplus \mathfrak{s o}(2,1)$ algebra. The motivation to use such basis is twofold. First, it allows us to recover the Maxwell CS gravity theory in a particular limit. Second, as we shall see, it reproduces the Maxwell field equations with a non-vanishing torsion.

A three-dimensional gravity can be formulated as a CS theory described by the action

$S_{\mathrm{CS}}[A]=\frac{k}{4 \pi} \int_{\mathcal{M}}\left\langle A \mathrm{~d} A+\frac{2}{3} A^{3}\right\rangle$,

with a given Lie algebra on a manifold $\mathcal{M}$, where $A$ is the gauge connection, $\langle$,$\rangle denotes the invariant trace and k=$ $1 /(4 G)$ is the CS level. For the sake of simplicity we have omitted writing the wedge product. The gauge connection one-form $A$ for the deformed Maxwell algebra reads

$A=e^{a} P_{a}+\omega^{a} J_{a}+f^{a} M_{a}$,

where $e^{a}, \omega^{a}$ and $f^{a}$ are the dreibein, the (dualized) spin connection and an auxiliary one-form field, respectively. The associated field strength $F=\mathrm{d} A+\frac{1}{2}[A, A]$ can be written as

$F=K^{a} P_{a}+R^{a} J_{a}+W^{a} M_{a}$,

where

$R^{a}=\mathrm{d} \omega^{a}+\frac{1}{2} \epsilon_{b c}^{a} \omega^{b} \omega^{c}$,

$K^{a}=T^{a}+\frac{1}{2 \ell} \epsilon_{b c}^{a} e^{b} e^{c}$,

$W^{a}=D(\omega) f^{a}+\frac{1}{2} \epsilon_{b c}^{a} e^{b} e^{c}$.

Here, $T^{a}=D(\omega) e^{a}$ is torsion two-form, $R^{a}$ is curvature two-form, and $D(\omega) \Phi^{a}=\mathrm{d} \Phi^{a}+\epsilon_{b c}^{a} \omega^{b} \Phi^{c}$ is the exterior covariant derivative. Naturally, the flat limit $\ell \rightarrow \infty$ reproduces the Maxwell field strength [29]. On the other hand, the non-degenerate bilinear form of the algebra (2.1) reads

$$
\begin{aligned}
& \left\langle J_{a} J_{b}\right\rangle=\alpha_{0} \eta_{a b}, \quad\left\langle P_{a} P_{b}\right\rangle=\left(\frac{\alpha_{1}}{\ell}+\alpha_{2}\right) \eta_{a b}, \\
& \left\langle J_{a} P_{b}\right\rangle=\alpha_{1} \eta_{a b}, \quad\left\langle P_{a} M_{b}\right\rangle=0, \\
& \left\langle J_{a} M_{b}\right\rangle=\alpha_{2} \eta_{a b}, \quad\left\langle M_{a} M_{b}\right\rangle=0,
\end{aligned}
$$

where $\alpha_{0}, \alpha_{1}$ and $\alpha_{2}$ are arbitrary constants satisfying $\alpha_{2} \neq 0$ and $\alpha_{1} \neq-\ell \alpha_{2}$. Both conditions are required to ensure the non-degeneracy of the invariant tensor (2.8). One can see that the flat limit $\ell \rightarrow \infty$ leads to the non-vanishing components of the invariant tensor for the Maxwell algebra [29].

Considering the one-form gauge potential (2.5) and the non-vanishing components of the invariant tensor (2.8), one can rewrite the CS action (2.4) as

$$
\begin{aligned}
S_{C S}= & \frac{k}{4 \pi} \int_{\mathcal{M}}\left\{\alpha_{0}\left(\omega^{a} \mathrm{~d} \omega_{a}+\frac{1}{3} \epsilon^{a b c} \omega_{a} \omega_{b} \omega_{c}\right)\right. \\
& +\alpha_{1}\left(2 R_{a} e^{a}+\frac{1}{3 \ell^{2}} \epsilon^{a b c} e_{a} e_{b} e_{c}+\frac{1}{\ell} T^{a} e_{a}\right) \\
& \left.+\alpha_{2}\left(T^{a} e_{a}+2 R^{a} f_{a}+\frac{1}{3 \ell} \epsilon^{a b c} e_{a} e_{b} e_{c}\right)\right\}
\end{aligned}
$$

up to a surface term. One can see that the CS action is proportional to three independent sectors each one with its respective coupling constant $\alpha_{i}$. In particular, the first term is the so-called exotic Lagrangian [2]. The second term contains the usual Einstein Lagrangian with cosmological constant term plus a torsional term related to the so-called Nieh-Yan invariant density.

It is interesting to notice that the CS gravity action (2.9) can be seen as a Maxwell extension of a particular case of the MB model, which describes a three-dimensional gravity model in presence of non-vanishing torsion. The MB action is given by

$I_{M B}=a I_{1}+\Lambda I_{2}+\beta_{3} I_{3}+\beta_{4} I_{4}$

where $a, \Lambda, \beta_{3}$ and $\beta_{4}$ are constants and

$I_{1}=2 \int e_{a} R^{a}$,

$I_{2}=-\frac{1}{3} \int \epsilon_{a b c} e^{a} e^{b} e^{c}$,

$I_{3}=\int \omega^{a} \mathrm{~d} \omega_{a}+\frac{1}{3} \epsilon^{a b c} \omega_{a} \omega_{b} \omega_{c}$,

$I_{4}=\int e_{a} T^{a}$.

Particularly, in the absence of the auxiliary field $f^{a}$ in (2.9), the constants appearing in the MB gravity can be identified with those of the deformed Maxwell algebra CS theory as

$$
\begin{gathered}
a=\frac{k}{4 \pi} \alpha_{1}, \quad \Lambda=-\frac{k}{4 \pi \ell}\left(\frac{\alpha_{1}}{\ell}+\alpha_{2}\right), \quad \beta_{3}=\frac{k}{4 \pi} \alpha_{0} \\
\beta_{4}=\frac{k}{4 \pi}\left(\frac{\alpha_{1}}{\ell}+\alpha_{2}\right) .
\end{gathered}
$$


Thus, the CS gravity action (2.9) can be interpreted as the Maxwellian version of a particular case of the MB gravity action when the MB's constants satisfy (2.12).

It is important to point out that one can accommodate a generalized cosmological constant in the Maxwell gravity theory using another deformation of the Maxwell algebra, known as AdS-Lorentz algebra [72]. However, in the AdSLorentz case, besides the Einstein-Hilbert term there is an additional gauge field $f_{a}$, while there is no torsion term $\alpha_{1}$ $[31,44,70,71]$. In the present case, the deformed Maxwell symmetry allows us to introduce both a cosmological constant and a torsion term. In the flat limit $\ell \rightarrow \infty$ the CS action reproduces the Maxwell CS gravity action which contains pure GR as sub-case. Dynamics of the $f_{a}$ gauge field is completely determined by the last term with coupling constant $\alpha_{2}$. In particular, the equations of motion appear by considering the variation of the action (2.9) under the respective gauge fields:

$\delta e^{a}: \quad 0=\alpha_{1}\left(R^{a}+\frac{1}{\ell} K^{a}\right)+\alpha_{2} K^{a}$,

$\delta \omega^{a}: \quad 0=\alpha_{0} R^{a}+\alpha_{1} K^{a}+\alpha_{2} W^{a}$,

$\delta f^{a}: \quad 0=\alpha_{2} R^{a}$.

Then, when $\alpha_{2} \neq 0$ we find the curvature two-forms (2.6) should vanish,

$R^{a}=0, \quad K^{a}=0, \quad W^{a}=0$.

Indeed, from last equation in (2.13), we find $R^{a}=0$. Nevertheless, it is important to emphasize that $\alpha_{1}=-\ell \alpha_{2}$, which solves the first equation of (2.13) and would imply a relation between $K^{a}$ and $W^{a}$, cannot be considered as a solution of the theory. As was previously mentioned, the non-degeneracy of the invariant tensor implies $\alpha_{2} \neq 0$ and $\alpha_{1} \neq-\ell \alpha_{2}$. In particular, in the three-dimensional CS formalism, the nondegeneracy of the bilinear form ensures that the CS action involves a kinematical term for each gauge field and the equations of motion imply that all curvature two-forms vanish as in (2.14). Note that the CS gravity theory (2.9) describes the Maxwell CS gravity theory in presence of a non-vanishing torsion $T^{a} \neq 0$. In particular, the first two equations $R^{a}=0$ and $T^{a}=-\frac{1}{2 \ell} \epsilon_{b c}^{a} e^{b} e^{c}$ correspond to the three-dimensional teleparallel theory in which the cosmological constant can be seen as a source for the torsion. On the other hand, the vanishing of $W^{a}$ implies that the exterior covariant derivative of the auxiliary field $f_{a}$ is constant. In particular, in the flat limit $\ell \rightarrow \infty$ the field equation for $f_{a}$ remains untouched and is analogue to the constancy of the electromagnetic field in flat spacetime.

One can see that each term of the action (2.9) is invariant under the gauge transformation laws of the algebra (2.1). Indeed, considering

$\Lambda=\varepsilon^{a} P_{a}+\rho^{a} J_{a}+\chi^{a} M_{a}$, we have that the gauge transformations $\delta A=\mathrm{d} \Lambda+[A, \Lambda]$ of the theory are given by

$$
\begin{aligned}
& \delta_{\Lambda} e^{a}=D(\omega) \varepsilon^{a}-\epsilon^{a b c} \rho_{b} e_{c}+\frac{1}{\ell} \epsilon^{a b c} e_{b} \varepsilon_{c}, \\
& \delta_{\Lambda} \omega^{a}=D(\omega) \rho^{a}, \\
& \delta_{\Lambda} f^{a}=D(\omega) \chi^{a}+\epsilon^{a b c} e_{b} \varepsilon_{c}-\epsilon^{a b c} \rho_{b} f_{c} .
\end{aligned}
$$

In this work, we analyze the consequences of this particular deformation of the Maxwell symmetry at the level of the asymptotic structure. In the Maxwell case, as was shown in [29], the presence of the additional gauge field $f^{a}$ leads to new effects compared to GR and the asymptotic symmetries is found to be a deformed $\mathfrak{b m s}_{3}$, denoted as $\mathfrak{M a x}_{3}$ in [66]. Here, we explore the implications of deforming the Maxwell algebra as in (2.1).

Let us recall that given an action there are two ways to render it having a well-posed variation principle. One of them is to add boundary terms to the action and the other is imposing suitable boundary conditions on fields. Let us consider the variation of the action (2.4),

$\delta S_{\mathrm{CS}}[A]=\frac{k}{2 \pi} \int_{\mathcal{M}}\langle\delta A F\rangle+\frac{k}{4 \pi} \int_{\partial \mathcal{M}}\langle\delta A A\rangle$,

where $\partial \mathcal{M}$ is the boundary of $\mathcal{M}$. The field equations require vanishing of the field strength and the on-shell boundary contribution to the action is the surface term

$\left.\delta S_{\mathrm{CS}}[A]\right|_{\text {bdy }}=-\frac{k}{4 \pi} \int_{\partial \mathcal{M}}\langle A \delta A\rangle$.

In our case this term reads as

$$
\begin{gathered}
\left.\delta S_{\mathrm{CS}}\right|_{\mathrm{bdy}}=\frac{k}{4 \pi} \int_{\partial \mathcal{M}}\left[\delta \omega^{a}\left(\alpha_{0} \omega_{a}+\alpha_{1} e_{a}+\alpha_{2} f_{a}\right)\right. \\
\left.+\delta e^{a}\left(\alpha_{1} \omega_{a}+\left(\frac{\alpha_{1}}{l}+\alpha_{2}\right) e_{a}\right)+\alpha_{2} \delta f^{a} \omega_{a}\right] .
\end{gathered}
$$

We will see later that for spacetimes with null boundary, where the boundary is located at $r=$ const $\rightarrow \infty$, the action principle is satisfied without addition of boundary terms.

\subsection{BMS-like solution}

In this section we analyze the field equations (2.14). We consider spacetimes with null boundary, which can be described in the three-dimensional BMS gauge. We parametrize spacetime by the local coordinates $x^{\mu}=(u, r, \phi)$, where $-\infty<$ $u<\infty$ is the retarded time coordinate, $\phi \sim \phi+2 \pi$ is the angular coordinate and the boundary is located at $r=$ const. Then, the metric can be written as [18]

$\mathrm{d} s^{2}=\mathcal{M} \mathrm{d} u^{2}-2 \mathrm{~d} u \mathrm{~d} r+\mathcal{N} \mathrm{d} \phi \mathrm{d} u+r^{2} \mathrm{~d} \phi^{2}$,

where $\mathcal{M}$ and $\mathcal{N}$ are two arbitrary functions of $u, \phi$. As was previously discussed, the deformed Maxwell symmetry (2.1) can be written in a certain basis as the direct sum $\mathfrak{i s o}(2,1) \oplus \mathfrak{s o}(2,1)$. Following the same trick considered in 
[31], we can find solutions of the present theory by first working in the direct sum basis and then, go to the basis we are interested in. In the direct sum basis, the fields $\left(\tilde{\omega}^{a}, \tilde{e}^{a}\right)$ associated to the $i \mathfrak{s o}(2,1)$ generators obey the very well-known GR boundary conditions, and the field $\tilde{f}^{a}$ associated to the $\mathfrak{s o}(2,1)$ generator can be set as a flat connection.

Furthermore, in the aforementioned direct sum basis, the functions $\mathcal{M}$ and $\mathcal{N}$ are given for the known results in asymptotically flat gravity in three dimensions

$\mathcal{M}=\mathcal{M}(\phi), \quad \mathcal{N}=\mathcal{J}(\phi)+u \mathcal{M}^{\prime}(\phi)$.

The spacetime line element can be written in terms of the dreibein as $\mathrm{d} s^{2}=\eta_{a b} \tilde{e}^{a} \tilde{e}^{b}$, where

$\eta_{a b}=\left(\begin{array}{lll}0 & 1 & 0 \\ 1 & 0 & 0 \\ 0 & 0 & 1\end{array}\right)$

is the Minkowski metric in null coordinate system. Then, the dreibein and the torsionless spin connection one-forms are written as

$\tilde{e}^{0}=-\mathrm{d} r+\frac{1}{2} \mathcal{M} \mathrm{d} u+\frac{1}{2} \mathcal{N} \mathrm{d} \phi, \quad \tilde{e}^{1}=\mathrm{d} u, \quad \tilde{e}^{2}=r \mathrm{~d} \phi$,

$\tilde{\omega}^{0}=\frac{1}{2} \mathcal{M d} \phi, \quad \tilde{\omega}^{1}=\mathrm{d} \phi, \quad \tilde{\omega}^{2}=0$.

The field $\tilde{f}^{a}$ can then be chosen as a Lorentz flat connection,

$\tilde{f}^{0}=\frac{1}{2} \mathcal{L} \mathrm{d} \phi, \quad \tilde{f}^{1}=\mathrm{d} \phi, \quad \tilde{f}^{2}=0$,

where $\mathcal{L}=\mathcal{L}(\phi)$. In this way, we have found the solutions in the BMS gauge for the fields $\left(\tilde{e}^{a}, \tilde{\omega}^{a}, \tilde{f}^{a}\right)$.

As mentioned before, we are interested in the basis where the Poincaré-Lorentz symmetry appears as a deformation of the Maxwell algebra. From (2.2), it is possible to show that the fields $\left(e^{a}, \omega^{a}, f^{a}\right)$ are related to those in the direct sum basis $\left(\tilde{e}^{a}, \tilde{\omega}^{a}, \tilde{f}^{a}\right)$ as follows:

$$
\begin{gathered}
e^{a}=\ell\left(\tilde{f}^{a}-\tilde{\omega}^{a}\right), \quad \omega^{a}=\tilde{\omega}^{a}, \\
f^{a}=\ell^{2}\left(\tilde{f}^{a}-\tilde{\omega}^{a}\right)-\ell \tilde{e}^{a} .
\end{gathered}
$$

Consequently, the field equations (2.14) are solved by the following components of the gauge fields

$e^{0}=\frac{1}{2} \mathcal{P} \mathrm{d} \phi, \quad \omega^{0}=\frac{1}{2} \mathcal{M} \mathrm{d} \phi, \quad f^{0}=\ell \mathrm{d} r+\frac{1}{2} \mathcal{F} \mathrm{d} \phi-\frac{\ell}{2} \mathcal{M} \mathrm{d} u$,

$e^{1}=0, \quad \omega^{1}=\mathrm{d} \phi, \quad f^{1}=-\ell \mathrm{d} u$,

$e^{2}=0, \quad \omega^{2}=0, \quad f^{2}=-\ell r \mathrm{~d} \phi$,

where, for later convenience, we have defined the functions $\mathcal{P}=\ell(\mathcal{L}-\mathcal{M})$ and $\mathcal{F}=\ell(\mathcal{P}-\mathcal{N})$.

As discussed we need to ensure vanishing of the boundary term in the variation of the action when suitable boundary conditions on the fields are imposed. The radial dependence of the connection $A$ can be gauged away by the gauge transformation $A=h^{-1} \mathrm{~d} h+h^{-1} a h$, where the asymptotic field $a=a_{u}(u, \phi) \mathrm{d} u+a_{\phi}(u, \phi) \mathrm{d} \phi$ does not depend on $r$ and $h=e^{\ell r M_{0}}$. Then, at the boundary $r=$ const. $\rightarrow \infty$, the on-shell action (2.18) takes the form

$$
\begin{aligned}
\left.\delta S_{\mathrm{CS}}\right|_{\mathrm{bdy}}= & -\frac{k}{4 \pi} \int_{\partial \mathcal{M}}\langle a \delta a\rangle \\
= & \frac{k}{4 \pi} \int_{\partial \mathcal{M}} \mathrm{d} u \mathrm{~d} \phi\left[\delta e_{u}^{a}\left(\alpha_{1} \omega_{a \phi}+\left(\frac{\alpha_{1}}{\ell}+\alpha_{2}\right) e_{a \phi}\right)\right. \\
& -\delta e_{\phi}^{a}\left(\alpha_{1} \omega_{a u}+\left(\frac{\alpha_{1}}{\ell}+\alpha_{2}\right) e_{a u}\right) \\
& +\delta \omega_{u}^{a}\left(\alpha_{0} \omega_{a \phi}+\alpha_{1} e_{a \phi}+\alpha_{2} f_{a \phi}\right) \\
& -\delta \omega_{\phi}^{a}\left(\alpha_{0} \omega_{a u}+\alpha_{1} e_{a u}+\alpha_{2} f_{a u}\right) \\
& \left.+\alpha_{2} \delta f_{u}^{a} \omega_{a \phi}-\alpha_{2} \delta f_{\phi}^{a} \omega_{a u}\right]
\end{aligned}
$$

Furthermore, from (2.26) we find the following boundary conditions for the gauge fields

$e_{u}^{a}=0, \quad \omega_{u}^{a}=0, \quad \omega_{\phi}^{a}=-\ell f_{u}^{a}$,

upon the first two, the variation of the action reduces to

$\left.\delta S_{\mathrm{CS}}\right|_{\mathrm{bdy}}=\frac{k \alpha_{2}}{4 \pi} \int_{\partial \mathcal{M}} \mathrm{d} u \mathrm{~d} \phi\left(\delta \omega_{\phi}^{a} f_{a u}-\delta f_{u}^{a} \omega_{a \phi}\right)$.

Finally, applying the last condition $\omega_{\phi}^{a}=-\ell f_{u}^{a}$, we arrive at

$\left.\delta S_{\mathrm{CS}}\right|_{\text {bdy }}=0$,

for any value of $\alpha_{2}$. Thus, in space-time with boundary conditions (2.28), the action principle is well-posed.

\section{Asymptotic symmetries}

The aim of this section is to find the asymptotic symmetry algebra for the Maxwell CS gravity with torsion which was previously constructed. To start with, we provide the suitable fall-off conditions for the gauge fields at infinity and the gauge transformations which preserve our boundary conditions. Then, the charge algebra is found using the ReggeTeitelboim method [73].

\subsection{Boundary conditions}

Inspired by the results obtained in the previous section, we consider the gauge connection evaluated in the BMS gauge as follows

$$
\begin{aligned}
A= & \frac{1}{2} \mathcal{M}(u, \phi) \mathrm{d} \phi J_{0}+\mathrm{d} \phi J_{1}+\frac{1}{2} \mathcal{P}(u, \phi) \mathrm{d} \phi P_{0} \\
& +\left[\ell \mathrm{d} r+\frac{1}{2} \mathcal{F}(u, \phi) \mathrm{d} \phi-\frac{\ell}{2} \mathcal{M}(u, \phi) \mathrm{d} u\right] M_{0} \\
& -\ell \mathrm{d} u M_{1}-r \ell \mathrm{d} \phi M_{2} .
\end{aligned}
$$


The radial dependence can be gauged away by an appropriate gauge transformation on the connection

$A=h^{-1} \mathrm{~d} h+h^{-1} a h$,

where the group element is given by $h=e^{\ell r M_{0}}$. Then, if we use the identity $h^{-1} \mathrm{~d} h=\ell \mathrm{d} r M_{0}$, and the Baker-CampbellHausdorff formula, we find

$h^{-1} a h=a-\ell r \mathrm{~d} \phi M_{2}$.

Therefore, once we have dropped out the radial dependence from the gauge field $A$, we are left with the asymptotic field $a=a_{u} \mathrm{~d} u+a_{\phi} \mathrm{d} \phi$, whose components are given by

$a_{u}=-\frac{\ell}{2} \mathcal{M} M_{0}-\ell M_{1}$,

$a_{\phi}=\frac{1}{2} \mathcal{M} J_{0}+J_{1}+\frac{1}{2} \mathcal{P} P_{0}+\frac{1}{2} \mathcal{F} M_{0}$,

which depend only on time and the angular coordinate. The equations of motion, which are required to hold in the asymptotic region, imply that

$\mathcal{M}=\mathcal{M}(\phi), \quad \mathcal{P}=\mathcal{P}(\phi), \quad \mathcal{F}=\mathcal{Z}(\phi)-u \ell \mathcal{M}^{\prime}(\phi)$.

\subsection{Residual gauge transformations}

Asymptotic symmetries correspond to residual gauge transformations $\delta_{\Lambda} A=\mathrm{d} \Lambda+[A, \Lambda]$ which preserve boundary conditions (3.1). We consider the following gauge parameters

$$
\begin{aligned}
\Lambda= & h^{-1} \lambda h, \quad \lambda=\lambda_{(J)}^{a}(u, \phi) J_{a} \\
& +\lambda_{(P)}^{a}(u, \phi) P_{a}+\lambda_{(M)}^{a}(u, \phi) M_{a} .
\end{aligned}
$$

Then, gauge transformations of the connection $A$ with gauge parameter $\Lambda$, lead to $r$-independent gauge transformations of the connection $a$ with gauge parameter $\lambda$, i.e.

$\delta_{\Lambda} a \equiv \delta_{\lambda} a=\mathrm{d} \lambda+[a, \lambda]$.

The gauge transformations that preserve the boundary conditions (3.1) with (3.5) for $\lambda_{(J)}^{a}$ and $\lambda_{(P)}^{a}$ are given by

$\lambda_{(J)}^{0}=\frac{\mathcal{M}}{2} \varepsilon-\varepsilon^{\prime \prime}, \quad \lambda_{(P)}^{0}=\frac{1}{2}\left[\mathcal{P}\left(\frac{\chi}{\ell}+\varepsilon\right)+\mathcal{M} \chi\right]-\chi^{\prime \prime}$,

$\lambda_{(J)}^{1}=\varepsilon, \quad \lambda_{(P)}^{1}=\chi$,

$\lambda_{(J)}^{2}=-\varepsilon^{\prime}, \quad \lambda_{(P)}^{2}=-\chi^{\prime}$,

while for $\lambda_{(M)}^{a}$ we get

$\lambda_{(M)}^{0}=\frac{\mathcal{M}}{2} \gamma+\frac{\mathcal{P}}{2} \chi+\frac{\mathcal{F}}{2} \varepsilon-\frac{\ell \mathcal{M}}{2} u \varepsilon^{\prime}+u \ell \varepsilon^{\prime \prime \prime}-\gamma^{\prime \prime}$,

$\lambda_{(M)}^{1}=\gamma-u \ell \varepsilon^{\prime}$,

$\lambda_{(M)}^{2}=-\gamma^{\prime}+u \ell \varepsilon^{\prime \prime}$, where $\varepsilon, \chi$ and $\gamma$ are three arbitrary, periodic functions of the angular coordinate $\phi$. Under the given gauge transformation, the dynamical fields transform as

$$
\begin{aligned}
\delta_{\lambda} \mathcal{M}= & \mathcal{M}^{\prime} \varepsilon+2 \mathcal{M} \varepsilon^{\prime}-2 \varepsilon^{\prime \prime \prime}, \\
\delta_{\lambda} \mathcal{P}= & \mathcal{P}^{\prime}\left(\frac{\chi}{\ell}+\varepsilon\right)+2 \mathcal{P}\left(\varepsilon^{\prime}+\frac{\chi^{\prime}}{\ell}\right) \\
& +\mathcal{M}^{\prime} \chi+2 \mathcal{M} \chi^{\prime}-2 \chi^{\prime \prime \prime}, \\
\delta_{\lambda} \mathcal{Z}= & \mathcal{Z}^{\prime} \varepsilon+2 \mathcal{Z} \varepsilon^{\prime}+\mathcal{M}^{\prime} \gamma \\
& +2 \mathcal{M} \gamma^{\prime}+\mathcal{P}^{\prime} \chi+2 \mathcal{P} \chi^{\prime}-2 \gamma^{\prime \prime \prime} .
\end{aligned}
$$

3.3 Canonical surface charges and asymptotic symmetry algebra

Asymptotic symmetries of the Maxwell gravity theory with non-vanishing torsion can be found in the canonical approach [73]. In particular, in the case of a three-dimensional ChernSimons theory, the variation of the canonical generators is given by $[74,75]$

$\delta Q[\lambda]=\int d \phi\left\langle\lambda \delta a_{\phi}\right\rangle$.

Therefore one can show that surface charge variation associated with (3.8) and (3.9) is

$\delta Q(\varepsilon, \chi, \gamma)=\int_{0}^{2 \pi} \mathrm{d} \phi(\varepsilon \delta \mathbf{J}+\chi \delta \mathbf{P}+\gamma \delta \mathbf{M})$

with

$$
\begin{aligned}
\mathbf{J} & =\frac{k}{4 \pi}\left(\alpha_{2} \mathcal{Z}+\alpha_{0} \mathcal{M}+\alpha_{1} \mathcal{P}\right), \\
\mathbf{P} & =\frac{k}{4 \pi}\left[\left(\frac{\alpha_{1}}{\ell}+\alpha_{2}\right) \mathcal{P}+\alpha_{1} \mathcal{M}\right], \\
\mathbf{M} & =\frac{k}{4 \pi} \alpha_{2} \mathcal{M} .
\end{aligned}
$$

One can take $\varepsilon, \chi$ and $\gamma$ to be state-independent and then the charge variation (3.12) is integrable on the phase space. There are three independent surface charges,

$$
\begin{aligned}
& J(\varepsilon)=Q(\varepsilon, 0,0), \quad P(\chi)=Q(0, \chi, 0), \\
& M(\gamma)=Q(0,0, \gamma),
\end{aligned}
$$

associated with three independent symmetry generators $\varepsilon, \chi$ and $\gamma$. It is shown that the algebra among surface charges is given by $[73,76,77]$

$\left\{Q\left(\Lambda_{1}\right), Q\left(\Lambda_{2}\right)\right\}=Q\left(\left[\Lambda_{1}, \Lambda_{2}\right]\right)+\mathcal{C}\left(\Lambda_{1}, \Lambda_{2}\right)$

where Dirac bracket is defined as $\left\{Q\left(\Lambda_{1}\right), Q\left(\Lambda_{2}\right)\right\}:=$ $\delta_{\Lambda_{2}} Q\left(\Lambda_{1}\right)$ and $\mathcal{C}\left(\Lambda_{1}, \Lambda_{2}\right)$ is central extension term. Therefore, using the transformation laws (3.10), one can show that 
the algebra of charges (3.12) is

$$
\begin{aligned}
&\left\{J\left(\varepsilon_{1}\right), J\left(\varepsilon_{2}\right)\right\}=J\left(\left[\varepsilon_{1}, \varepsilon_{2}\right]\right)-\frac{k \alpha_{0}}{2 \pi} \int \mathrm{d} \phi \varepsilon_{1} \varepsilon_{2}^{\prime \prime \prime}, \\
&\{J(\varepsilon), P(\chi)\}=P([\varepsilon, \chi])-\frac{k \alpha_{1}}{2 \pi} \int \mathrm{d} \phi \varepsilon \chi^{\prime \prime \prime}, \\
&\{J(\varepsilon), M(\gamma)\}=M([\varepsilon, \gamma])-\frac{k \alpha_{2}}{2 \pi} \int \mathrm{d} \phi \varepsilon \gamma^{\prime \prime \prime}, \\
&\left\{P\left(\chi_{1}\right), P\left(\chi_{2}\right)\right\}=M\left(\left[\chi_{1}, \chi_{2}\right]\right)+\frac{1}{\ell} P\left(\left[\chi_{1}, \chi_{2}\right]\right) \\
&\{P(\chi), M(\gamma)\}=0, \\
&\left\{M\left(\gamma_{1}\right), M\left(\gamma_{2}\right)\right\}=0,
\end{aligned}
$$

where $[x, y]=x y^{\prime}-y x^{\prime}$. The resulting algebra corresponds to an infinite-dimensional lift of the deformed Maxwell algebra. Now we can express the above algebra in Fourier modes,

$$
J_{n}:=J\left(e^{i n \phi}\right), \quad P_{n}:=P\left(e^{i n \phi}\right), \quad M_{n}:=M\left(e^{i n \phi}\right),
$$

which give rise to the following centrally extended algebra

$$
\begin{aligned}
i\left\{J_{m}, J_{n}\right\}= & (m-n) J_{m+n}+\frac{c_{1}}{12} m^{3} \delta_{m+n, 0}, \\
i\left\{J_{m}, P_{n}\right\}= & (m-n) P_{m+n}+\frac{c_{2}}{12} m^{3} \delta_{m+n, 0}, \\
i\left\{J_{m}, M_{n}\right\}= & (m-n) M_{m+n}+\frac{c_{3}}{12} m^{3} \delta_{m+n, 0}, \\
i\left\{P_{m}, P_{n}\right\}= & (m-n) M_{m+n}+\frac{1}{\ell}(m-n) P_{m+n} \\
& +\frac{1}{12}\left(\frac{c_{2}}{\ell}+c_{3}\right) m^{3} \delta_{m+n, 0}, \\
i\left\{P_{m}, M_{n}\right\}= & 0, \\
i\left\{M_{m}, M_{n}\right\}= & 0 .
\end{aligned}
$$

The central charges $c_{1}, c_{2}$ and $c_{3}$ are related to the CS level $k$ and the arbitrary constant appearing in the invariant tensor (2.8) as

$c_{i}=12 k \alpha_{i-1}$

\subsection{Change of basis}

The infinite-dimensional algebra (3.18) can be seen as the infinite-dimensional enhancement of the deformed Maxwell algebra (2.1). In particular, in the flat limit $\ell \rightarrow \infty$ we recover the asymptotic symmetry of the three-dimensional Maxwell CS gravity theory introduced in [29]. Interestingly, as was shown in [66], the algebra (3.18) is isomorphic to the $\widehat{\mathfrak{b m s}_{3}} \oplus$ $\mathfrak{v i r}$ algebra. To observe this at the level of charge algebra, we use the change of basis proposed in [78].

Suppose $\varepsilon, \chi$ and $\gamma$ are now state-dependent, i.e. they are functions of dynamical fields. We require that charge variation be integrable which leads to

$\varepsilon=\frac{\delta \mathcal{G}}{\delta \mathbf{J}}, \quad \chi=\frac{\delta \mathcal{G}}{\delta \mathbf{P}}, \quad \gamma=\frac{\delta \mathcal{G}}{\delta \mathbf{M}}$,

for some functional

$\mathcal{G}[\mathbf{J}, \mathbf{P}, \mathbf{M}]=\int_{0}^{2 \pi} \mathrm{d} \phi \mathbf{G}(\mathbf{J}, \mathbf{P}, \mathbf{M})$.

By choosing

$\mathbf{G}=\tilde{\varepsilon}\left(\mathbf{J}-\ell \mathbf{P}-\ell^{2} \mathbf{M}\right)+\tilde{\chi}\left(\ell \mathbf{P}+\ell^{2} \mathbf{M}\right)-\ell \tilde{\gamma} \mathbf{M}$

where $\tilde{\varepsilon}, \tilde{\chi}, \tilde{\gamma}$ are state-independent functions, one can show that the charge variation (3.12) can be written as

$\delta Q(\tilde{\varepsilon}, \tilde{\chi}, \tilde{\gamma})=\int_{0}^{2 \pi} \mathrm{d} \phi(\tilde{\varepsilon} \delta \mathbf{L}+\tilde{\chi} \delta \mathbf{S}+\tilde{\gamma} \delta \mathbf{T})$

with

$\mathbf{L}=\mathbf{J}-\ell \mathbf{P}-\ell^{2} \mathbf{M}$,

$\mathbf{S}=\ell \mathbf{P}+\ell^{2} \mathbf{M}$

$\mathbf{T}=-\ell \mathbf{M}$.

Now, by introducing Fourier modes

$$
\begin{aligned}
L_{m} & :=Q\left(\tilde{\varepsilon}=e^{i n \phi}, 0,0\right), \quad S_{m}:=Q\left(0, \tilde{\chi}=e^{i n \phi}, 0\right), \\
T_{m} & :=Q\left(0,0, \tilde{\gamma}=e^{i n \phi}\right),
\end{aligned}
$$

one finds the direct sum of the $\widehat{\mathfrak{b m s}_{3}}$ and Virasoro algebra:

$$
\begin{aligned}
& i\left\{L_{m}, L_{n}\right\}=(m-n) L_{m+n}+\frac{c_{L L}}{12} m^{3} \delta_{m+n, 0}, \\
& i\left\{L_{m}, T_{n}\right\}=(m-n) T_{m+n}+\frac{c_{L T}}{12} m^{3} \delta_{m+n, 0}, \\
& i\left\{S_{m}, S_{n}\right\}=(m-n) S_{m+n}+\frac{c_{S S}}{12} m^{3} \delta_{m+n, 0},
\end{aligned}
$$

where the central charges are related to those appearing in (3.18) as

$c_{L L} \equiv c_{1}-\ell c_{2}-\ell^{2} c_{3}, \quad c_{S S} \equiv \ell c_{2}+\ell^{2} c_{3}, \quad c_{L T} \equiv-\ell c_{3}$.

The central charges $\left(c_{L L}, c_{L T}, c_{S S}\right)$ of the $\widehat{\mathfrak{b m s}_{3}} \oplus$ vir algebra are related to the three independent terms of the CS gravity action for the $\mathfrak{i s o}(2,1) \oplus \mathfrak{s o}(2,1)$ algebra.

Let us note that the algebra (3.18) can be obtained alternatively as a central extension of the deformed $\mathfrak{M a x}_{3}[66]$ and deformed $\mathfrak{b m s}_{4}$ [67]. It is also worth pointing out that by ignoring the generators $T_{m}$ and central charge $c_{L T}$ in (3.26), which is equivalent to ignoring the Maxwell generators $M_{m}$ and central charge $c_{3}$ in (3.18), one obtains two copies of Virasoro algebra, which is the asymptotic symmetry of the $\mathrm{AdS}_{3} \mathrm{CS}$ gravity (or Teleparallel theory [79]) with two central charges as

$c_{L L} \equiv c_{1}-\ell c_{2}, \quad c_{S S} \equiv \ell c_{2}$. 


\section{Discussion and outlook}

In this work, we have studied a CS gravity theory with a deformed Maxwell algebra as a gauge group, which allows us to introduce a non-vanishing torsion to the Maxwell CS gravity. In particular, the CS action can be seen as a Maxwell generalization of a particular case of the MB gravity [54] whose field equations correspond to those of the Maxwell gravity theory but in presence of a non-vanishing torsion. Motivated by the fact that the deformed Maxwell is isomorphic to the $\mathfrak{i s o}(2,1) \oplus \mathfrak{s o}(2,1)$ algebra, we considered asymptotically flat geometries and discuss the BMS-like solution. We then explored the implications of the deformed Maxwell algebra (2.1) at the level of the asymptotic symmetry. In particular, we have shown that the asymptotic symmetry for the Maxwell algebra with torsion is described by an infinite-enhancement of the deformed Maxwell algebra which can be written as the $\widehat{\mathfrak{b m s}_{3}} \oplus \mathfrak{v i r}$ algebra with three independent central charges.

It would be interesting to go further in the study of the solutions of the present theory. In particular, one expects the solutions to describe a Maxwell version of the so-called Teleparallel theory in which the cosmological constant is a source for the torsion. As was shown in [57,79], both GR with cosmological constant and three-dimensional Teleparallel gravity have the same asymptotic symmetry and dynamics. Furthermore, three-dimensional gravity with torsion possesses BTZ [80] black hole solution $[56,81,82]$ whose thermodynamics properties have been discussed in [59]. Then, it seems natural to expect to find a BTZ type solution for our theory. The approach to this last point and thermodynamics remains as an interesting open issue to explore. In particular, one can study the effects of the present deformation in the vacuum energy and vacuum angular momentum of the stationary configuration and their relations to the GR and Maxwell ones.

Although the three-dimensional Maxwell algebra can be deformed into $\mathfrak{s o}(2,2) \oplus \mathfrak{s o}(2,1)$ and $\mathfrak{i s o}(2,1) \oplus \mathfrak{s o}(2,1)$ algebras, the former can be obtained as a deformation of the latter. The same argument is true for their corresponding infinite enhancements, meaning that the $\mathfrak{b m s}_{3} \oplus \mathfrak{w i t t}$ algebra can be deformed into the $\mathfrak{w i t t} \oplus \mathfrak{w i t t} \oplus$ witt algebra. Since both infinite-dimensional algebras are obtained as asymptotic symmetry algebras of different three-dimensional CS gravity theories, it might be interesting to consider the deformation relation between these theories at the level of their solutions, phase spaces and physical quantities. It should be pointed out that the $\mathfrak{b m s}_{3} \oplus \mathfrak{w i t t}$ algebra can also be deformed into another algebra which is known as $W(a, b) \oplus$ witt, as was proved in [30]. Regarding the recent work [78], where it was shown that the $W(0, b)$ algebra can be obtained as near horizon symmetries of three-dimensional black holes, a natural question that one can ask is what is the interpretation of the
$W(a, b) \oplus \mathfrak{w i t t}$ algebra in the context of the new CS theory. It would be worthwhile to explore the answer.

Another aspect that deserves to be explored is supersymmetric extension of our analysis and results. Supersymmetric extensions of three-dimensional gravity with torsion has been constructed in $[61,83]$. On the other hand, the threedimensional CS supergravity invariant under the Maxwell algebra has been recently presented in [45,49-51]. However, to our knowledge, a supersymmetric Maxwell CS gravity with torsion has not been discussed yet. It would be then interesting to explore diverse supersymmetric extensions of the deformed Maxwell algebra and study which superalgebra is a good candidate to construct a well-defined supersymmetric gravity with torsion in three spacetime dimensions. The study of supersymmetric extensions of CS gravity with torsion and their asymptotic structures could bring a better understanding of the role of torsion.

Acknowledgements The authors would like to thank N. Merino and M. M. Sheikh-Jabbari for the correspondence and comments. This work was funded by the National Agency for Research and Development (ANID) CONICYT - PAI grant No. 77190078 (P.C.) and FONDECYT Project No. 3170438 (E.R.). H.A. and H.R.S. acknowledge the partial support of Iranian NSF under grant no. 950124. H.A. acknowledges the support by the Saramadan grant no. ISEF/M/98204. H.R.S. wishes to thank to M. Henneaux and S. Detournay for their kind hospitality at the Physique Théorique et Mathématique department of the Université Libre de Bruxelles (ULB) which part of this work was done and acknowledges the support by F.R.S.-FNRS fellowship "Bourse de séjour scientifique IN". P.C. would like to thank to the Dirección de Investigación and Vice-rectoria de Investigación of the Universidad Católica de la Santísima Concepción, Chile, for their constant support.

Data Availability Statement This manuscript has no associated data or the data will not be deposited. [Authors' comment: The results obtained and presented in the manuscript did not require any data.]

Open Access This article is licensed under a Creative Commons Attribution 4.0 International License, which permits use, sharing, adaptation, distribution and reproduction in any medium or format, as long as you give appropriate credit to the original author(s) and the source, provide a link to the Creative Commons licence, and indicate if changes were made. The images or other third party material in this article are included in the article's Creative Commons licence, unless indicated otherwise in a credit line to the material. If material is not included in the article's Creative Commons licence and your intended use is not permitted by statutory regulation or exceeds the permitted use, you will need to obtain permission directly from the copyright holder. To view a copy of this licence, visit http://creativecomm ons.org/licenses/by/4.0/.

Funded by $\mathrm{SCOAP}^{3}$.

\section{References}

1. A. Achucarro, P. Townsend, A Chern-Simons action for threedimensional anti-de Sitter supergravity theories. Phys. Lett. B 180, 89 (1986)

2. E. Witten, $(2+1)$-Dimensional gravity as an exactly soluble system. Nucl. Phys. B 311, 46 (1988) 
3. J. Zanelli, Lecture notes on Chern-Simons (super-)gravities. Second edition (February 2008), in Geometric and Topological Methods for Quantum Field Theory. Proceedings, Summer School, Villa de Leyva, Colombia, July 9-27, 2001 (2005), p. 2. arXiv:hep-th/0502193

4. J.D. Brown, M. Henneaux, Central charges in the canonical realization of asymptotic symmetries: an example from three dimensional gravity. Commun. Math. Phys. 104(2), 207-226 (1986)

5. H. Bondi, M.G.J. van der Burg, A.W.K. Metzner, Gravitational waves in general relativity. 7. Waves from axisymmetric isolated systems. Proc. R. Soc. Lond. A 269, 21-52 (1962)

6. R. Sachs, Asymptotic symmetries in gravitational theory. Phys. Rev. 128, 2851-2864 (1962)

7. A. Ashtekar, J. Bičák, B.G. Schmidt, Asymptotic structure of symmetry reduced general relativity. Phys. Rev. D 55, 669-686 (1997). arXiv:gr-qc/9608042

8. G. Barnich, C. Troessaert, Aspects of the BMS/CFT correspondence. JHEP 05, 062 (2010). arXiv:1001.1541

9. G. Barnich, G. Compère, Classical central extension for asymptotic symmetries at null infinity in three spacetime dimensions. Class. Quantum Gravity 24(5), F15 (2007)

10. J.M. Maldacena, The large N limit of superconformal field theories and supergravity. Int. J. Theor. Phys. 38, 1113-1133 (1999). arXiv:hep-th/9711200

11. M. Henneaux, L. Maoz, A. Schwimmer, Asymptotic dynamics and asymptotic symmetries of three-dimensional extended AdS supergravity. Ann. Phys. 282, 31-66 (2000). arXiv:hep-th/9910013

12. M. Henneaux, C. Martinez, R. Troncoso, Asymptotically anti-de Sitter spacetimes in topologically massive gravity. Phys. Rev. D 79, 081502 (2009). arXiv:0901.2874

13. K. Skenderis, M. Taylor, B.C. van Rees, Topologically massive gravity and the AdS/CFT correspondence. JHEP 09, 045 (2009). arXiv:0906.4926

14. H. Afshar, B. Cvetkovic, S. Ertl, D. Grumiller, N. Johansson, Conformal Chern-Simons holography-lock, stock and barrel. Phys. Rev. D 85, 064033 (2012). arXiv: 1110.5644

15. G. Compère, W. Song, A. Strominger, New boundary conditions for $\mathrm{AdS}_{3}$. JHEP 05, 152 (2013). arXiv: 1303.2662

16. C. Troessaert, Enhanced asymptotic symmetry algebra of $\mathrm{AdS}_{3}$. JHEP 08, 044 (2013). arXiv:1303.3296

17. H.A. Gonzalez, M. Pino, Boundary dynamics of asymptotically flat 3D gravity coupled to higher spin fields. JHEP 05, 127 (2014). arXiv: 1403.4898

18. G. Barnich, L. Donnay, J. Matulich, R. Troncoso, Asymptotic symmetries and dynamics of three-dimensional flat supergravity. JHEP 08, 071 (2014). arXiv: 1407.4275

19. O. Fuentealba, J. Matulich, R. Troncoso, Extension of the Poincaré group with half-integer spin generators: hypergravity and beyond. JHEP 09, 003 (2015). arXiv: 1505.06173

20. O. Fuentealba, J. Matulich, R. Troncoso, Asymptotically flat structure of hypergravity in three spacetime dimensions. JHEP 10, 009 (2015). arXiv: 1508.04663

21. A. Pérez, D. Tempo, R. Troncoso, Boundary conditions for General Relativity on $\mathrm{AdS}_{3}$ and the KdV hierarchy. JHEP 06, 103 (2016). arXiv: 1605.04490

22. D. Grumiller, M. Riegler, Most general $\mathrm{AdS}_{3}$ boundary conditions. JHEP 10, 023 (2016). arXiv: 1608.01308

23. N. Banerjee, D.P. Jatkar, I. Lodato, S. Mukhi, T. Neogi, Extended supersymmetric $\mathrm{BMS}_{3}$ algebras and their free field realisations. JHEP 11, 059 (2016). arXiv: 1609.09210

24. I. Lodato, W. Merbis, Super-BMS 3 algebras from $\mathcal{N}=2$ flat supergravities. JHEP 11, 150 (2016). arXiv:1610.07506

25. S. Detournay, M. Riegler, Enhanced asymptotic symmetry algebra of $2+1$ dimensional flat space. Phys. Rev. D 95(4), 046008 (2017). arXiv: 1612.00278
26. O. Fuentealba, J. Matulich, R. Troncoso, Asymptotic structure of $\mathcal{N}=2$ supergravity in $3 \mathrm{D}$ : extended super-BMS 3 and nonlinear energy bounds. JHEP 09, 030 (2017). arXiv:1706.07542

27. N. Banerjee, I. Lodato, T. Neogi (2017) $N=4$ Supersymmetric BMS3 algebras from asymptotic symmetry analysis. Phys. Rev D 96(6), 066029. arXiv: 1706.02922

28. R. Caroca, P. Concha, E. Rodríguez, P. Salgado-Rebolledo, Generalizing the $\mathfrak{b m s}_{3}$ and 2D-conformal algebras by expanding the Virasoro algebra. Eur. Phys. J. C 78(3), 262 (2018). arXiv:1707.07209

29. P. Concha, N. Merino, O. Miskovic, E. Rodríguez, P. SalgadoRebolledo, O. Valdivia, Asymptotic symmetries of threedimensional Chern-Simons gravity for the Maxwell algebra. JHEP 10, 079 (2018). arXiv:1805.08834

30. A. Farahmand Parsa, H.R. Safari, M.M. Sheikh-Jabbari, On rigidity of 3d asymptotic symmetry algebras. JHEP 03, 143 (2019). arXiv: 1809.08209

31. P. Concha, N. Merino, E. Rodríguez, P. Salgado-Rebolledo, O. Valdivia, Semi-simple enlargement of the $\mathfrak{b m s}_{3}$ algebra from a $\mathfrak{s o}(2,2) \oplus \mathfrak{s o}(2,1)$ Chern-Simons theory. JHEP 02, 002 (2019). arXiv: 1810.12256

32. R. Caroca, P. Concha, O. Fierro, E. Rodríguez, Three-dimensional Poincaré supergravity and $N$-extended supersymmetric $B M S_{3}$ algebra. Phys. Lett. B 792, 93-100 (2019). arXiv:1812.05065

33. N. Banerjee, A. Bhattacharjee, Neetu, T. Neogi, New $\mathrm{N}=2$ SuperBMS $_{3}$ algebra and invariant dual theory for 3D supergravity. JHEP 11, 122 (2019). arXiv:1905.10239

34. R. Caroca, P. Concha, O. Fierro, E. Rodríguez, On the supersymmetric extension of asymptotic symmetries in three spacetime dimensions. Eur. Phys. J. C 80(1), 29 (2020). arXiv:1908.09150

35. R. Schrader, The Maxwell group and the quantum theory of particles in classical homogeneous electromagnetic fields. Fortschritte der Physik 20(12), 701-734 (1972)

36. H. Bacry, P. Combe, J. Richard, Group-theoretical analysis of elementary particles in an external electromagnetic field II.-The nonrelativistic particle in a constant and uniform field. Il Nuovo Cimento A (1965-1970) 70(3), 289-312 (1970)

37. J. Gomis, A. Kleinschmidt, On free Lie algebras and particles in electro-magnetic fields. JHEP 07, 085 (2017). arXiv:1705.05854

38. J.D. Edelstein, M. Hassaine, R. Troncoso, J. Zanelli, Liealgebra expansions, Chern-Simons theories and the EinsteinHilbert Lagrangian. Phys. Lett. B 640, 278-284 (2006). arXiv:hep-th/0605174

39. F. Izaurieta, E. Rodriguez, P. Minning, P. Salgado, A. Perez, Standard general relativity from Chern-Simons gravity. Phys. Lett. B 678, 213-217 (2009). arXiv:0905.2187

40. P.K. Concha, D.M. Peñafiel, E.K. Rodríguez, P. Salgado, Evendimensional general relativity from Born-Infeld gravity. Phys. Lett. B 725, 419-424 (2013). arXiv:1309.0062

41. P.K. Concha, D.M. Penafiel, E.K. Rodriguez, P. Salgado, ChernSimons and Born-Infeld gravity theories and Maxwell algebras type. Eur. Phys. J. C 74, 2741 (2014). arXiv:1402.0023

42. P.K. Concha, D.M. Peñafiel, E.K. Rodríguez, P. Salgado, Generalized Poincaré algebras and Lovelock-Cartan gravity theory. Phys. Lett. B 742, 310-316 (2015). arXiv:1405.7078

43. P. Salgado, R.J. Szabo, O. Valdivia, Topological gravity and transgression holography. Phys. Rev. D 89(8), 084077 (2014). arXiv: 1401.3653

44. S. Hoseinzadeh, A. Rezaei-Aghdam, $(2+1)$-Dimensional gravity from Maxwell and semisimple extension of the Poincaré gauge symmetric models. Phys. Rev. D 90(8), 084008 (2014). arXiv: 1402.0320

45. S. Hoseinzadeh, A. Rezaei-Aghdam, $(2+1)$-Dimensional gravity from Maxwell and semisimple extension of the Poincare gauge symmetric models. Phys. Rev. D 90(8), 084008 (2014). arXiv: 1402.0320 
46. R. Caroca, P. Concha, O. Fierro, E. Rodríguez, P. SalgadoRebolledo, Generalized Chern-Simons higher-spin gravity theories in three dimensions. Nucl. Phys. B 934, 240-264 (2018). arXiv: 1712.09975

47. L. Avilés, E. Frodden, J. Gomis, D. Hidalgo, J. Zanelli, Nonrelativistic Maxwell Chern-Simons gravity. JHEP 05, 047 (2018). arXiv: 1802.08453

48. S. Bansal, D. Sorokin, Can Chern-Simons or Rarita-Schwinger be a Volkov-Akulov Goldstone? JHEP 07, 106 (2018). arXiv: 1806.05945

49. P. Concha, D.M. Peñafiel, E. Rodríguez, On the Maxwell supergravity and flat limit in $2+1$ dimensions. Phys. Lett. B 785, 247253 (2018). arXiv: 1807.00194

50. P. Concha, N-extended Maxwell supergravities as Chern-Simons theories in three spacetime dimensions. Phys. Lett. B 792, 290-297 (2019). arXiv:1903.03081

51. P. Concha, L. Ravera, E. Rodríguez, Three-dimensional Maxwellian extended Bargmann supergravity. JHEP 04, 051 (2020). arXiv: 1912.09477

52. D. Chernyavsky, N.S. Deger, D. Sorokin, Spontaneously broken $3 d$ Hietarinta-Maxwell Chern-Simons theory and minimal massive gravity. Eur. Phys. J. C 80, 556 (2020). arXiv:2002.07592

53. P. Concha, M. Ipinza, E. Rodríguez, Generalized Maxwellian exotic Bargmann gravity theory in three spacetime dimensions. Phys. Lett. B 807, 135593 (2020). arXiv:2004.01203

54. E.W. Mielke, P. Baekler, Topological gauge model of gravity with torsion. Phys. Lett. A 156, 399-403 (1991)

55. P. Baekler, E. Mielke, F. Hehl, Dynamical symmetries in topological 3-D gravity with torsion. Nuovo Cim. B 107, 91-110 (1992)

56. M. Blagojevic, M. Vasilic, 3-D gravity with torsion as a Chern-Simons gauge theory. Phys. Rev. D 68, 104023 (2003). arXiv:gr-qc/0307078

57. M. Blagojevic, M. Vasilic, Asymptotic dynamics in 3-D gravity with torsion. Phys. Rev. D 68, 124007 (2003). arXiv:gr-qc/0306070

58. S.L. Cacciatori, M.M. Caldarelli, A. Giacomini, D. Klemm, D.S. Mansi, Chern-Simons formulation of three-dimensional gravity with torsion and nonmetricity. J. Geom. Phys. 56, 2523-2543 (2006). arXiv:hep-th/0507200

59. M. Blagojevic, B. Cvetkovic, Black hole entropy in 3-D gravity with torsion. Class. Quantum Gravity 23, 4781 (2006). arXiv:gr-qc/0601006

60. M. Blagojevic, B. Cvetkovic, Black hole entropy from the boundary conformal structure in 3D gravity with torsion. JHEP 10, 005 (2006). arXiv:gr-qc/0606086

61. A. Giacomini, R. Troncoso, S. Willison, Three-dimensional supergravity reloaded. Class. Quantum Gravity 24, 2845-2860 (2007). arXiv:hep-th/0610077

62. D. Klemm, G. Tagliabue, The CFT dual of AdS gravity with torsion. Class. Quantum Gravity 25, 035011 (2008). arXiv:0705.3320

63. R.C. Santamaria, J.D. Edelstein, A. Garbarz, G.E. Giribet, On the addition of torsion to chiral gravity. Phys. Rev. D 83, 124032 (2011). arXiv:1102.4649

64. B. Cvetković, D. Simić, Near-horizon geometry with torsion. Phys. Rev. D 99(2), 024032 (2019). arXiv: 1809.00555

65. J. Peleteiro, C. Valcárcel, Spin-3 Mielke-Baekler gravity. Class. Quant. Grav. 37, 185010 (2020). arXiv:2003.02627
66. P. Concha, H. Safari, On stabilization of Maxwell-BMS algebra. JHEP 04, 073 (2020). arXiv:1909.12827

67. H.R. Safari, M.M. Sheikh-Jabbari, BMS 4 algebra, its stability and deformations. JHEP 04, 068 (2019). arXiv: 1902.03260

68. J. Hietarinta, Supersymmetry generators of arbitrary spin. Phys. Rev. D 13, 838 (1976)

69. J. Gomis, K. Kamimura, J. Lukierski, Deformations of Maxwell algebra and their dynamical realizations. JHEP 08, 039 (2009). arXiv:0906.4464

70. J. Diaz, O. Fierro, F. Izaurieta, N. Merino, E. Rodriguez, P. Salgado, O. Valdivia, A generalized action for $(2+1)$-dimensional ChernSimons gravity. J. Phys. A 45, 255207 (2012). arXiv:1311.2215

71. P. Concha, E. Rodríguez, Non-relativistic gravity theory based on an enlargement of the extended Bargmann algebra. JHEP 07, 085 (2019). arXiv: 1906.00086

72. D.V. Soroka, V.A. Soroka, Tensor extension of the Poincaré algebra. Phys. Lett. B 607, 302-305 (2005). arXiv:hep-th/0410012

73. T. Regge, C. Teitelboim, Role of surface integrals in the Hamiltonian formulation of general relativity. Ann. Phys. 88(1), 286-318 (1974)

74. M. Banados, Three-dimensional quantum geometry and black holes. AIP Conf. Proc. 484(1), 147-169 (1999). arXiv:hep-th/9901148

75. M. Banados, Global charges in Chern-Simons field theory and the $(2+1)$ black hole. Phys. Rev. D 52, 5816-5825 (1996). arXiv:hep-th/9405171

76. J.D. Brown, M. Henneaux, On the Poisson brackets of differentiable generators in classical field theory. J. Math. Phys. 27(2), 489-491 (1986)

77. G. Compère, Advanced Lectures on General Relativity, vol. 952 (Springer, Berlin, 2019)

78. D. Grumiller, A. Pérez, M.M. Sheikh-Jabbari, R. Troncoso, C. Zwikel, Spacetime structure near generic horizons and soft hair. Phys. Rev. Lett. 124, 041601 (2020). arXiv:1908.09833

79. M. Blagojevic, M. Vasilic, Asymptotic symmetries in 3-d gravity with torsion. Phys. Rev. D 67, 084032 (2003). arXiv:gr-qc/0301051

80. M. Banados, C. Teitelboim, J. Zanelli, The Black hole in threedimensional space-time. Phys. Rev. Lett. 69, 1849-1851 (1992). arXiv:hep-th/9204099

81. A.A. Garcia, F.W. Hehl, C. Heinicke, A. Macias, Exact vacuum solution of a $(1+2)$-dimensional Poincaré gauge theory: BTZ solution with torsion. Phys. Rev. D 67, 124016 (2003). arXiv:gr-qc/0302097

82. E.W. Mielke, A.A. Rincon Maggiolo, Rotating black hole solution in a generalized topological 3-D gravity with torsion. Phys. Rev. D 68, $104026(2003)$

83. B. Cvetkovic, M. Blagojevic, Supersymmetric 3D gravity with torsion: asymptotic symmetries. Class. Quantum Gravity 24, 3933 3950 (2007). arXiv:gr-qc/0702121 